\title{
The relationship between the immune system and oral manifestations of inflammatory bowel disease: a review
}

\author{
MIROSLAV VASOVIC ${ }^{1}$, NEVENA GAJOVIC ${ }^{2}$, DENIS BRAJKOVIC ${ }^{1}$, MARINA JOVANOVIC ${ }^{3}$, \\ NATASA ZDRAVKOVAIC ${ }^{3}$, TATJANA KANJEVAC ${ }^{l}$ \\ 'Department of Dentistry, Faculty of Medical Sciences, University of Kragujevac, Serbia \\ ${ }^{2}$ Center for Molecular Medicine and Stem Cells Research, Faculty of Medical Sciences, University of Kragujevac, Serbia \\ ${ }^{3}$ Department of Internal Medicine, Faculty of Medical Sciences, University of Kragujevac, Serbia
}

\begin{abstract}
Inflammatory bowel diseases (IBDs) are chronic, relapsing inflammatory diseases characterized by exacerbations and remissions of the gastrointestinal tract, clinically manifested as Crohn's disease and ulcerative colitis. The etiology of IBDs is considered to be multi factorial, comprising environmental, immune, microbial and genetic factors. Clinical signs may include abdominal pain, frequent bloody diarrheas, mucorrhea, vomiting, fever, fatigue or weight loss. Changes in the oral cavity often precede intestinal symptoms. Inflammatory bowel disease leads to a significant deterioration of oral health, which indicates that cooperation between the dentist and the gastroenterologist is necessary when considering patients' welfare. Patients with IBD have an altered immune response, but microorganisms of the oral cavity may also be responsible for its modification.

This review paper discusses the correlation between the immune system and inflammatory bowel disease manifestations in the oral cavity.
\end{abstract}

Key words: oral disease, oral health, dental management, inflammatory bowel diseases, immune system.

(Cent Eur J Immunol 2016; (3): 302-310)

\section{Introduction}

Inflammatory bowel diseases (IBDs) are a group of chronic, relapsing inflammatory diseases of gastrointestinal tract (GIT) with various extra-intestinal symptoms $[1,2]$. The etiology of IBDs is considered to be multi factorial, comprising environmental, immune, microbial and genetic factors [3-5]. Males and females are equally affected by IBDs, with the onset of disease occurring in most cases between the second and third decade of life $[1,2]$. Diagnosis of IBDs is based on the correlation of clinical, radiological, endoscopic and histopathological findings [6]. IBDs are clinically manifested as Crohn's disease (CD) and ulcerative colitis (UC).

Ulcerative colitis is a chronic intestinal inflammatory disease characterized by ulcerations of the colonic superficial lining mucosa and submucosa, without affecting deeper layers of the intestinal wall [2]. Ulcerations present features of active disease followed by frequent bloody diarrheas, mucorrhea and abdominal pain as well as the systemic signs: fever, fatigue, weight loss, anemia and sepsis [1, 2].
Crohn's disease, unlike UC, may affect any part of GIT and it is characterized by transmural inflammatory lesions affecting the terminal ileum (50\% of the cases), colon (30\%), ileo-colonic (20\%) [1, 2]. While UC has continuous distribution throughout the colon, $\mathrm{CD}$ has patchy distribution, leading to stenosis at different levels of GIT, which may be complicated with penetration of the intestinal wall (fistulas, abscesses) [1, 2]. Clinical symptoms may vary depending on localization, disease activity and type (stricturing or penetrating). Clinical signs may include abdominal pain, diarrhea, vomiting, fever, fatigue or weight loss [1, 2].

The objective of this review paper is to summarize current knowledge concerning the relationship between the immune system and inflammatory bowel disease manifestations in the oral cavity in order to improve dental treatment options.

\section{Immunological background of IBD}

The immune system seems to play an important role in the development and progression of IBDs [7]. Leukocytes

Correspondence: Tatjana Kanjevac, Department of Dentistry, Faculty of Medical Sciences, University of Kragujevac, Svetozara Markovica 69, 34000 Kragujevac, Serbia, e-mail: stomatologija@medf.kg.ac.rs

Submitted: 10.07.2015; Accepted: 28.07.2015 
are increased and activated in intestinal lamina propria of IBDs patients and in several colitis models [8]. The immune system is made up of innate and acquired immunity. Innate immunity is composed of neutrophils, dendritic cells, NK cells, and monocyte/macrophages while acquired immunity consists of B lymphocytes (humoral immunity) and $\mathrm{T}$ lymphocytes (cellular immunity) [9]. T lymphocytes can be further divided between $\mathrm{CD}^{+}$helper and $\mathrm{CD} 8^{+}$cytotoxic T lymphocytes [9]. The major property of $\mathrm{CD}^{+}$ helper $\mathrm{T}$ lymphocytes is the production of cytokines and, according to the type of cytokines they produce, $\mathrm{CD}^{+}$ $\mathrm{T}$ lymphocytes can be classified into Th-1 (IFN- $\gamma$ and TNF- $\alpha$ ), Th-2 (IL-4, IL-5 and IL-13), Th-17 (IL-6, IL-17, IL-22) and Tregs (IL-10 and TGF- $\beta$ ) (Fig. 1) [9].

In light of the critical role of $\mathrm{CD}^{+}$lymphocytes in IBDs pathogenesis, further elucidation of $\mathrm{CD}^{+}$cellderived cytokine regulatory networks would greatly facilitate the understanding of the disease and the development of novel therapeutic strategies [8].

In our recent study, we have found significantly higher serum levels of both pro- and anti-inflammatory cytokines: TNF- $\alpha$, TGF- $\beta$, IL-4, IL-6, IL-10, IFN- $\gamma$ in patients with UC compared to healthy controls [10]. Analysis of the contra-regulatory cytokines ratio revealed significantly higher values of IFN- $\gamma / \mathrm{IL}-4$, IFN- $\gamma / \mathrm{TGF}-\beta$, IFN- $\gamma / \mathrm{IL}-10$, IL-17/ IL-10, IL-6/TGF- $\beta$, IL-27/IL-17, IL-17/TGF- $\beta$ ratio in patients with UC compared to healthy controls, indicating pro-inflammatory cytokine predominance in patients with UC [10]. According to available data, the increased expression of pro-inflammatory cytokines, such as TNF- $\alpha, \mathrm{IL}-1 \beta$ and IL-6, and a deficiency of immunosuppressive IL-10 are hallmarks of intestinal inflammation [11-13]. However, UC is characterized by its Th- 2 cytokine pattern, while CD is considered a Th-1 disease.

Pro-inflammatory cytokine IL-6 plays a key role in the induction and development of intestinal inflammation [1416]. Our previous results showed higher levels of IL-6 and CRP in sera of patients with UC and a correlation with the presence of extra-intestinal manifestations of disease [10]. In line with this finding, other study results showed that IL-6 was the predominant cytokine found in inflamed areas of UC patients and its concentration correlated with the Mayo endoscopic score for severity of the disease [10]. IL-6 is produced mainly by monocytes/macrophages and plays a major role in the development of the Th-17 immune response [17]. IL- 6 and TGF- $\beta$ direct the differentiation of naive Th lymphocytes towards the Th-17 phenotype [17-21]. We found higher values of IL- 6 and IL-17/IL-10 ratio in patients with extra-intestinal manifestations of UC, while TGF- $\beta$ values were unchanged, suggesting a key role of IL- 6 in the development of the Th-17 immune response [10]. The increased expression of IL-17 in intestinal mucosa and its increased concentration in serum of patients with UC suggest that Th-17 cells play an important role in UC [10]. Other study results show that the number of Th-17 cells and expressions of IL-17, IL-21 and IL-22 were significantly increased in intestinal tissue biopsies taken from patients with active UC and with active $\mathrm{CD}$ in comparison to healthy controls [22]. Th-17 lymphocytes, a subpopulation of T helper lymphocytes, were isolated for the first time from the peripheral blood of patients with $\mathrm{CD}$ as the subclass of lymphocytes that produce IL-17 and IL-23 [23]. Abnormal activity of innate and acquired immune cells that produce IL-17 has been implicated in the pathogenesis of autoimmune and inflammatory diseases, including CD. Distinct mucosal profiles of cytokines are produced during the different phases of CD [24]. In mucosal samples with established lesions, there was a mixed Th- $1 /$ Th- 17 response with no TNF- $\alpha$ induction. The expression of IL-4 and IL-5 was up-regulated in both early and established lesions even though the fraction of IL-4 producing cells was lower than that of cells producing either IFN- $\gamma$ or IL-17A [24]. Recent study results have revealed that $\mathrm{CD} 14^{+} \mathrm{CD} 163^{\text {low }}$ cells from inflamed regions of mucosa of patients with CD expressed high levels of IL-6, IL-23p19, and TNF- $\alpha$ mRNAs, and strongly induced Th-17 cell development [25]. CD14+ CD163 ${ }^{\text {low }}$ cells from non-inflamed mucosa of patients with $\mathrm{CD}$ also had increased abilities to induce Th-17 cells, compared with those from normal intestinal mucosa [25].

The increased concentration of TNF- $\alpha$ in sera of patients with CD suggests a Th- 1 immune response [26-28]. TNF- $\alpha$ induces production of IL- 6 , followed by a higher level of IL-6 in sera of patients with CD. In patients with active CD, salivary IL-1 $\beta$, IL-6, and TNF- $\alpha$ levels were higher than in patients with inactive disease and controls. Elevated salivary IL-6 and TNF- $\alpha$ levels correlate with specific oral lesions [29]. Results of our study have shown higher concentrations of TNF- $\alpha$ and IL- 6 as well as levels of IL-6/TGF- $\beta$ and IL-17/TGF- $\beta$ ratios in patients with CD compared to patients with UC, indicating a predominance of pro-inflammatory Th- 1 and Th-17 immune response in CD (summarized in Table 1) [10]. The others have confirmed that the Th-1 immune response and production of IL-2, IL-8, IL-12, IFN- $\gamma$ and TNF- $\alpha$ were predominant in CD [26].

The predominance of the Th- 1 immune response, manifested by significantly lower serum levels of anti-inflammatory IL-10 and a significantly higher IFN- $\gamma / \mathrm{IL}-10$ ratio, was detected in patients with complications of UC [10]. Furthermore, a positive correlation of the serum level of TNF- $\alpha$ with the histological grade of disease, and the higher concentration of CRP detected in patients with active UC, confirmed the development of the Th-1 immune response in patients with severe disease [10]. Recent studies have also found a positive correlation of sera CRP with clinical activity of disease and TNF- $\alpha$ with inflammation and histological grade of inflamed mucosa in patients with UC [14, 30]. The increased production of pro-inflammatory TNF- $\alpha$ can induce an intensive inflammatory response with enhanced migration of leukocytes and subsequent damage to local tissue. A decreased concentration of the anti-inflammatory 
Table 1. Immune response properties in inflammatory bowel diseases

\begin{tabular}{ll}
\hline & \multicolumn{2}{l}{ Ulcerative colitis } \\
\hline$\uparrow$ IL-6 & Intestinal inflammation \\
\cline { 2 - 2 } & Extra-intestinal manifestations of disease \\
\hline$\uparrow$ IL-17/IL-10 ratio & Extra-intestinal manifestations of disease \\
\hline$\uparrow$ IFN- $\gamma /$ IL-10 ratio & Complications of disease \\
\hline$\uparrow$ TNF- $\alpha$ & Higher histological grade of disease \\
\hline$\downarrow$ IL-10 & Extra-intestinal manifestations of disease \\
\hline Ulcerative colitis & Crohn's disease \\
\hline$\uparrow$ TNF- $\alpha$ & + \\
\hline$\uparrow$ IL-6 & + \\
\hline$\uparrow$ IL-2 & + \\
\hline$\uparrow$ IL-8 & + \\
\hline$\uparrow$ IL-12 & + \\
\hline$\uparrow$ IFN- $\gamma$ & + \\
\hline$\uparrow$ IL-6/TGF- $\beta$ ratio & + \\
\hline$\uparrow$ IL-17/TGF- $\beta$ ratio & + \\
\hline
\end{tabular}

cytokine IL-10 was detected in patients with UC and with diagnosed extra-intestinal manifestations [10]. Others found a lower expression of IL-10 in colonic mucosa in patients with UC [31]. We also noticed lower values of IL-10 and the IFN- $\gamma / \mathrm{IL}-10$ ratio in patients with UC and the presence of complications such as stricture and pseudopolyposis, indicating the predominance of the Th-1 immune response [10]. It appears that a relatively lower level of IL-10 in some patients with UC is not enough to suppress the production of pro-inflammatory cytokines such as IL-6, TNF- $\alpha$, IFN- $\gamma$ and IL-17. These patients usually have severe disease. A higher IL-17/IL-10 ratio can represent an important hallmark of disease severity and progression, based on a correlation with intestinal and extra-intestinal manifestations [10].

The role of Th- 1 and Th-17 lymphocytes in the pathogenesis of CD is well established [32,33], while UC seems to be predominantly a Th-2 disease [34]. Presented data, in line with recent findings, suggest a mixed profile rather than a Th- 2 cytokine profile in severe UC and disease progression can be indicated by an altered cytokine milieu (Fig. 1).

\section{Oral manifestations of IBDs}

IBDs are characterized by the co-existence of various extra-intestinal manifestations which often precede GIT symptoms $[1,2,6]$. Because of the rapid rise of incidence and prevalence of IBD, the systemic nature of the disease, limited treatment options and increased morbidity of the patients, IBD concerns diverse groups of medical and dental specialists. The oral health of patients with IBD is
Table 2. Orofacial symptoms associated with inflammatory bowel disease (numbers in the square brackets indicate ordinal numbers of the references)

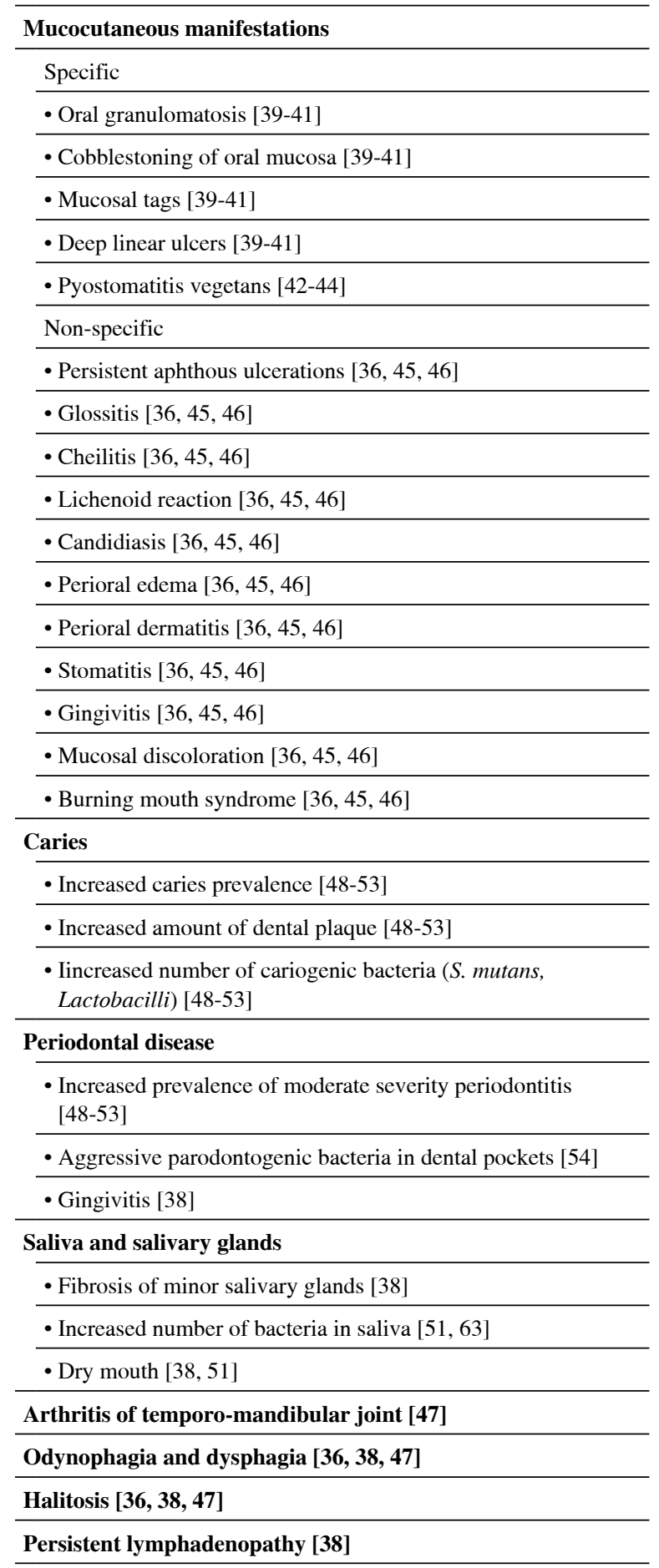

significantly deteriorated and a variety of different oral manifestations have been described (Table 2).

The oral symptoms of CD precede the intestinal manifestations in $60 \%$ of patients, and in many cases lead to 


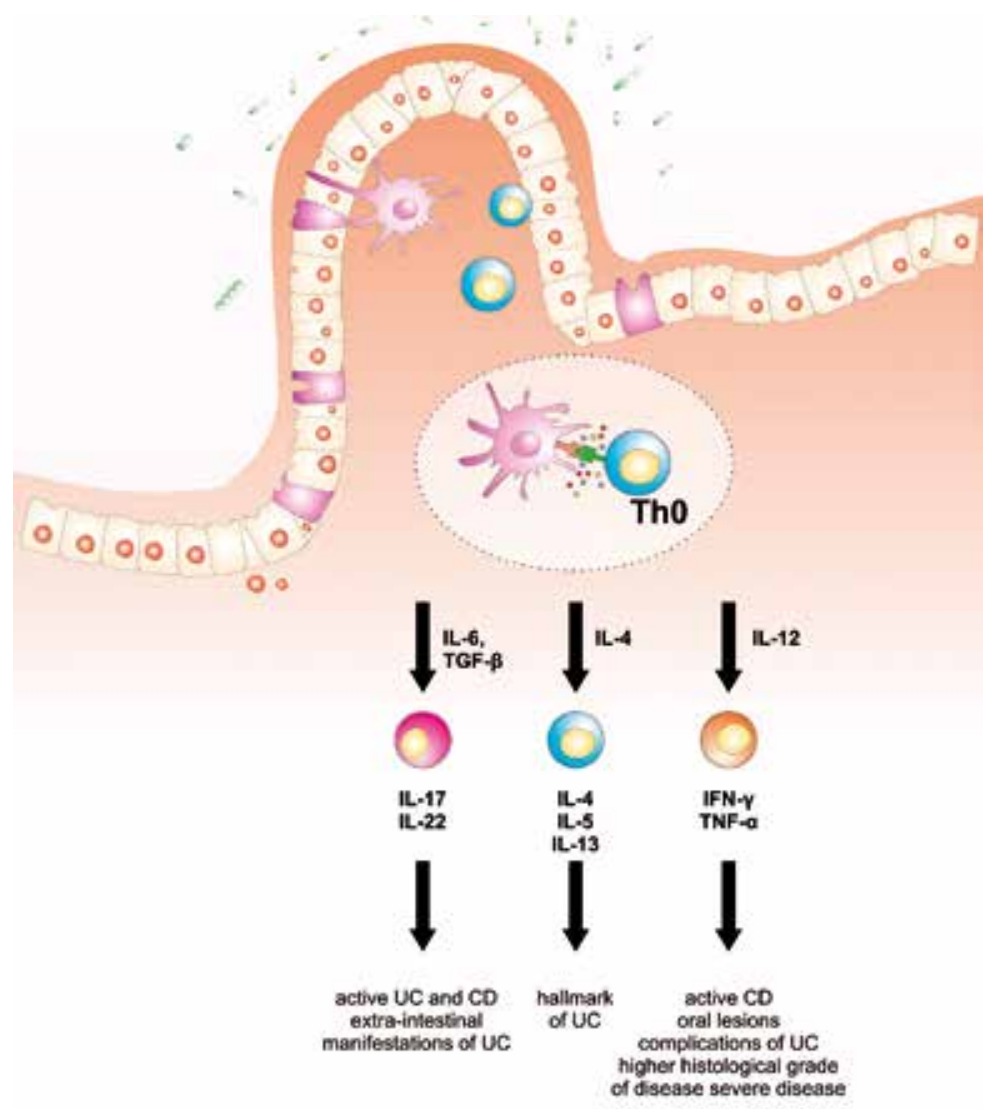

Fig. 1. Immune responses in the gut. During active inflammation, under stimulation of different cytokines, nadive CD4 ${ }^{+}$ helper T lymphocytes (Th0) differentiate into: Th1 (IFN- $\gamma$ and TNF- $\alpha$ ), Th2 (IL-4, IL-5 and IL-13) or Th17 (IL-17, IL-22) T cells. Different subsets of Th cells play various roles in disease immuno-pathogenesis and subsequent tissue destruction and systemic manifestations

diagnosis of the disease [35, 36]. Furthermore, oral manifestations often coincide with other extra-intestinal manifestations such as erythema nodosum and arthritis [37].

Clinical distinction between CD and UC with regard to the intraoral changes is uncertain. Specific oral manifestations are a result of the direct extension of intestinal inflammation of CD or the metastatic spread of CD and UC, with macroscopic changes similar to changes observed in the intestine [36-38]. Pathognomonic oral manifestations related to $\mathrm{CD}$ include oral granulomatosis, macrocheilia, cobblestoning of oral mucosa, mucosal tags and deep linear ulcers [39-41]. Pyostomatitis vegetans is more often seen in UC patients and reflects the active phase of the disease [42-44].

Non-specific oral lesions may be a result of malnutrition and malabsorption syndrome as well as of the drug therapy and include stomatitis, glossitis, aphthous ulcerations, cheilitis, perioral dermatitis, lichenoid reactions and candidiasis $[36,45,46]$. Aphthous ulcerations in patients with IBDs are more extensive and persistent and it is believed that they are exacerbated by IBDs and its management [47].
The occurrence of oral manifestations in patients with active IBDs may be a result of altered cytokine activity in entire GIT, including oral cavity. Szczeklik et al. have found elevated levels of pro-inflammatory cytokines IL-6, IL-1 $\beta$ and TNF- $\alpha$ in the saliva of patients with active CD along with manifestation of the specific oral lesions [48]. Similarly, Nielsen et al. demonstrated elevated levels of IL-6 in the saliva of CD patients [49]. Levels of pro-inflammatory cytokines IL-6, IL-1 $\beta$ and TNF- $\alpha$ are found to be elevated in the saliva of patients with different oral pathologies: periodontal disease, lichen planus and oral carcinoma [50, 51]. Furthermore, Rezaie et al. established significantly reduced antioxidant capacity of the saliva in patients with active CD [52]. Thus, levels of pro-inflammatory cytokines and parameters of oxidative stress in the saliva may be useful biomarkers of IBDs, particularly CD activity.

Many authors who reviewed the oral health of IBDs patients had found a higher prevalence of caries and periodontal disease than in age- and gender-matched controls without IBDs [53-58]. Grössner-Schreiber et al. found oral health to be significantly deteriorated in patients with IBD 
due to significantly more oral lesions, higher caries prevalence, more dental plaque and more generalized periodontal disease than matched controls [53]. The authors concluded that the extent, but not severity of periodontitis was significantly higher in IBDs patients than the control group, due to more sites of clinical attachment level (CAL) $\geq 4 \mathrm{~mm}(81 \%)$ and $\geq 5 \mathrm{~mm}$ (64\%). In contrast, Habashneh et al. found a positive correlation between periodontitis and $\mathrm{UC}$ and $\mathrm{CD}$, and both severity and extent of periodontitis were greater in UC and CD patients than in matched controls [55]. Brito et al. found more CAL $\geq 3 \mathrm{~mm}$ and more subjects with periodontitis than present in matched controls [54]. Interestingly, the extent of periodontal disease in this study was greater in UC patients than CD patients, which may be attributed to different immuno-pathogenesis of the diseases. Importantly, a high number of periodontopathogenic bacteria such as Campylobacter rectus, Porphyromonas gingivalis and Tannerella forsythia were found in the gingival sulcus of IBDs patients [59].

Periodontal disease and IBDs are characterized by chronic inflammation and share a number of similar pathophysiological features [60]. Similar to IBDs, periodontal disease is a chronic relapsing inflammatory disease of periodontal tissues. Its etiology is multi-factorial, and periodontopathogenic bacteria altering the immune response play a major role in pathogenesis [61]. The destruction of periodontal tissues is modified with the activation of various cytokines (IL-1, IL-6, TNF- $\alpha$ ) and abnormal oxidative stress similarly to the IBDs pathogenesis [62-65]. Unlike IBDs, where non-specific intestinal microorganisms trigger the immune system, periodontal disease is triggered by a specific group of microorganisms possessing virulent factors [61]. Another difference in the pathogenesis of the diseases is that the immune response in periodontal disease is B cell dependent, whilst the pathogenic mechanisms of IBD are T cell related [61]. Figuerede et al. have shown that the expression pattern of cytokines IL-6, IL-10, IL-18, TNF- $\alpha$, IFN- $\gamma$ was similar in the gingival crevicular fluid (GCF) of CD, UC and the control patients, all with chronic untreated periodontitis [66]. However in this study, the GCF level of anti-inflammatory and anti-apoptotic cytokine IL-4 in UC and CD patients was significantly lower compared to the controls. Salvi et al. have shown low levels of IL-4 in GCF to be associated with increased periodontal destruction [67]. Therefore, the GCF level of IL-4 could be a useful biomarker of periodontitis in IBDs patients. Whether the periodontal disease in IBDs patients is a symptom of IBDs or whether the higher prevalence of periodontal disease in IBDs patients is related to other oral manifestations could be a topic of future studies.

Caries prevalence in patients with IBDs is significantly increased compared to healthy controls [53, 57]. Established risk factors for dental caries in IBDs patients include an increased number of Lactobacilli and Streptococcus mutans, reduced salivary secretion, poor oral hygiene, increased sugar consumption and malabsorption [53-58]. The dietary habits of patients with IBDs include an increased intake of refined carbohydrates and sweetened drinks, especially for patients with acute exacerbations of the disease [57, 68, 69]. Patients with IBDs eat more frequently and take a smaller amount of food to avoid gastrointestinal difficulties [54]. DMFT was especially high among patients who have had an extensive small bowel resection, suggesting caries incidence could be related to malabsorption syndrome [70]. A prospective study by Sundh and Emilson investigated the salivary conditions and the dental status of CD patients [56]. The authors found that the saliva flow rate and the buffering capacity is normal with CD patients but the number of salivary S. mutans and Lactobacilli was high during the 3-year observation period.

Oral bacteria have been associated with systemic diseases, such as infective endocarditis, rheumatoid arthritis or pulmonary diseases [71-74]. Oral bacteria are able to reach the circulation and cause bacteremia following dental procedures such as tooth extraction, pocket curettage or even tooth polishing [73].

Recent studies have shown both cariogenic (S. mutans) and periodontogenic oral bacteria (Fusobacterium nucleatum, Campylobacter concisus) are associated with IBD symptoms [75-77]. Fusobacterium nucleatum strains found in intestinal biopsy tissues of IBDs patients were significantly more invasive than those isolated from control patients [76]. Enteric invasive $C$. concisus oral strains were detected in $50 \%$ of IBDs patients, and no healthy controls [77]. The link between a specific strain of $S$. mutans and UC has been studied recently. Serotype $k S$. mutans was connected with bacterial endocarditis and cerebral stroke as well as attenuation of symptoms of UC [78, 79]. According to the study by Ayoki et al., the specific strain of $k$ serotype $S$. mutans, TW 295, caused the attenuation of UC symptoms after bacteremia on a dextran sodium-sulphate induced mouse colitis model [80]. The authors hypothesized that elevated levels of IFN- $\gamma$ in GIT wall induced after colonization of hepatocytes by $S$. mutans, contributed to UC symptoms aggravation. In this study, the level of bacteremia was similar to bacteremia found after ordinary dental procedures [73]. Bearing in mind that bacteremia is associated with simple dental procedures and S. mutans is the most common oral bacteria detected in the blood samples, this finding could be of clinical relevance, and future studies are needed to clarify the association between other pathogenic oral bacteria and IBDs.

IBDs are associated with systemic bone loss and osteoporosis affecting about $4-60 \%$ of CD patients and $18 \%$ of UC patients [81]. Studies on chemically induced colitis on rats have shown decreased bone formation and increased bone turnover, which is essential for implant osseointegration $[82,83]$. Thus, IBDs present a high risk of early dental implant failure [84-86]. The risk factor for osteoporosis 
in IBDs include malabsorption syndrome, hypocalcemia, hypovitaminosis D and long-term immunosuppressive therapy [81]. A recent study showed that Klotho protein, an anti-inflammatory protein significant for bone mineral homeostasis, is reduced in an IBDs animal model [87].

\section{Recent advances in treatment of IBD patients}

The treatment of patients with chronic IBDs should reduce inflammation and to keep periods of remission as long as possible. The choice of treatment depends on the frequency of exacerbation periods, the scope and the severity of disease, and the presence of extraintestinal manifestations. The ideal treatment should control inflammation efficiently but, it is not supposed to cause the increased immunosuppression nor to produce adverse effects. There are two different therapeutic approaches to patients with IBDs: step up and top-down [88]. The first therapeutic approach refers to the conventional therapy and involves the use of aminosalicylates, antibiotics, corticosteroids, thiopurines and folic acid antagonists. Aminosalicylates are the first-line drugs for the treatment of UC. However, poor responses to the treatment as well as side effects limit their use. The use of metronidazole in patients with CD leads to a better condition of patients. Also, the use of ciprofloxacin reduces the severity of the disease. But, antibiotics are not enough to establish the balance between bad and good intestinal microorganisms, and in that case the use of probiotics is recommended. In the acute stages of the disease, corticosteroids are used. However, if they are used on a daily basis or for a long time, even in small doses, primarily systemic, they can cause numerous adverse effects [89]. The other therapeutic approach, top-down, is increasingly being used for the patients with considerable risk factors for severe inflammation or unfavorable course of the disease. It aims to stop the inflammatory process as early as possible and to prevent the occurrence of complications [90]. This therapeutic approach refers to the biological therapy, aimed at the development of agents that modulate cytokine signaling pathways.

Tumor necrosis factor $\alpha$ (TNF- $\alpha$ ) plays an important role in the pathogenesis of IBDs [10, 24, 26, 30]. TNF- $\alpha$ inhibition results in down-regulation of progressive inflammation, resulting in rapid and sustained clinical remission. Monoclonal antibodies targeting TNF- $\alpha$ are frequently used to treat IBDs [91]. For now, five TNF- $\alpha$ inhibitors (infliximab, etanercept, adalimumab, golimumab and certolizumab pegol) have been approved for treatment of immune-mediated inflammatory diseases [92]. Among them, adalimumab has the widest range of indications, showing its excellent efficacy and tolerability. The major limitation on the use of anti-TNF- $\alpha$ antibodies is that patients can develop tuberculosis soon after the initiation of treatment, due to TNF- $\alpha$ neutralization [93].
The role of IL-17 in genesis and development of IBDs is also well established [10,32,33]. Some studies show that administration of anti-IL-17A monoclonal antibody to patients with moderate to severe CD had no therapeutic effect, and in some patients acute exacerbation of disease was noted, suggesting a protective role of IL-17A [94]. A possible explanation of this controversial finding is that inhibition of IL-17A leads to an increase in Th-1 immune response.

Antibodies targeting IL-12/IL-23 pathways, and pro-inflammatory cytokines such as IFN- $\gamma$, IL- 2 and IL- 6 often show an initial promising result, but for none of these agents efficacy has unequivocally been established [95].

Anti-inflammatory cytokine IL-10 can suppress the exacerbated mucosal immune response and maintain intestinal homeostasis and commensal microbiota tolerance [70]. Several human trials have been undertaken using IL-10 in therapy of IBDs. The first trial conducted by van Deventer et al. has shown that IL-10 supplementation is safe and well tolerated [96]. This has been confirmed by subsequent studies [97, 98]. Meanwhile, some studies show that parenteral IL-10 treatment does not result in significantly reduced remission rates or clinical improvements, probably because of side effects of pharmacokinetics and tissue distribution $[80,99,100]$. IL-10 administration is only successful when administered prior to initiation of colitis and it is unable to treat any established inflammation. Therefore, IL-10 supplementation could be used to prevent relapses rather than to treat active inflammation [70, 101]. However, mucosal delivery of IL-10 protein or cDNA by nanoparticles or genetically modified microorganisms is becoming a promising and viable treatment option $[88,102,103]$.

\section{Dental care of IBD patients}

To the best of our knowledge, there is no uniform algorithm for dental care of IBD patients. The high incidence of caries, periodontitis and various orofacial mucosal and mucocutaneous manifestations of IBD imply the need for collaboration between different medical and dental specialists. Oral manifestations often precede the onset of intestinal symptoms, and therefore dentists should be familiar with the pathognomonic signs of IBD. Orofacial granulomatosis is particularly common in children presenting with $\mathrm{CD}$ and should be considered as an early symptom preceding GIT changes [87]. Patients with IBD should be referred for frequent dental checkups and periodontal evaluation. The importance of proper oral hygiene must be emphasized to IBD patients due to the fact that it is usually neglected because of the disrupted general health [104]. Dental treatment of IBD patients is frequently altered considering the limited use of non-steroidal antiinflammatory drugs, antibiotics or systemic corticosteroids and gastroenterologists should be consulted prior to their administration. Oral manifestations are often seen in the active phase of the disease and are successfully treated with topical corticosteroids or immunosuppressive drugs (thalidomide, 
tacrolimus), but these should not be used indefinitely due to the risk of mucosal atrophy [105]. Extensive dental procedures requiring administration of systemic corticosteroids and antibiotics need collaboration between dentists and gastroenterologists. The use of paracetamol is recommended, but may also cause adverse events [47]. Since bacteremia following dental procedures may extend IBDs symptoms and cause disease exacerbation, antibiotic prophylaxis for IBD patients prior to dental management may be beneficial [106].

\section{Conclusions}

Oral manifestations often precede the onset of intestinal symptoms in IBDs patients, and the dentist should suspect IBDs if pathognomonic oral manifestations are present. The significant deterioration of oral health in IBDs patients implies the need for frequent dental check-ups and periodontal evaluation of these patients. Similarly, collaboration between dentists and gastroenterological specialists is necessary, as dental treatment of IBDs patients should be modified according to disease activity and medication used. The mechanism of altered immune response in the intestinal wall in IBDs resembles the chronic inflammatory processes of periodontal disease. Bacteremia following simple dental procedures may carry a potential risk for an increase in IBDs symptoms throughout IFN- $\gamma$ mediated activation of intestinal inflammation. Future studies are needed to clarify the connection between immunological response in patients with IBDs and associated oral manifestations.

\section{The authors declare no conflict of interest.}

\section{References}

1. Baumgart DC, Sandborn WJ (2012): Crohn's disease. Lancet 380: 1590-1605.

2. Podolsky DK (2002): Inflammatory Bowel Disease. N Engl J Med 347: 417-429.

3. Dogan B, Scherl E, Bosworth B, et al. (2013): Multidrug resistance is common in Escherichia coli associated with ileal Crohn's disease. Inflamm Bowel Dis 19: 141-150.

4. Cho JH, Brant SR (2011): Recent Insights into the Genetics of Inflammatory Bowel Disease. Gastroenterology 140: 17041712.

5. Marks DJ, Rahman FZ, Sewell GW, Segal AW (2010): Crohn's disease: An immune deficiency state. Clin Rev Allergy Immunol 38: 20-31.

6. Trikudanathan G, Venkatesh PG, Navaneethan U (2012): Diagnosis and therapeutic management of extra-intestinal manifestations of inflammatory bowel disease. Drugs 72: 2333-2349.

7. Raza A, Yousaf W, Giannella R, Shata MT (2012): Th17 cells: interactions with predisposing factors in the immunopathogenesis of inflammatory bowel disease. Expert Rev Clin Immunol 8: 161-168.

8. Kappeler A, Mueller C (2000): The role of activated cytotoxic $\mathrm{T}$ cells in inflammatory bowel disease. Histol Histopathol 15 : 167-172.
9. Abbas AK, Lichtman AH, Pillai S (2012): Cellular and molecular immunology. 7 ed. Elsevier, Philadelphia; 225-243.

10. Zdravkovic ND, Jovanovic IP, Radosavljevic GD, et al. (2014): Potential Dual Immunomodulatory Role of VEGF in Ulcerative Colitis and Colorectal Carcinoma. Int J Med Sci 11: 936-947.

11. Hyun JG, Mayer L (2006): Mechanisms underlying inflammatory bowel disease. Drug Discov Today Dis Mech 3: 457-462.

12. Shih DQ, Targan SR (2008): Immunopathogenesis of inflammatory bowel disease. World J Gastroenterol 126: 1620-1633.

13. Li MC, He SH (2004): IL-10 and its related cytokines for treatment of inflammatory bowel disease. World J Gastroenterol 10: 620-625.

14. Murata Y, Ishiguro Y, Itoh J (1995): The role of proinflammatory and immunoregulatory cytokines in the pathogenesis of ulcerative colitis. J Gastroenterol 30: 56-60.

15. Atreya R, Neurath MF (2005): Involvement of IL-6 in the pathogenesis of inflammatory bowel disease and colon cancer. Clin Rev Allergy Immunol 28: 187-195.

16. Bernardo D, Vallejo-Díez S, Mann ER, et al. (2012): IL-6 promotes immune responses in human ulcerative colitis and induces a skin-homing phenotype in the dendritic cells and T cells they stimulate. Eur J Immunol 42: 1337-1353.

17. Oukka M (2007): Interplay between pathogenic Th17 and regulatory T cells. Ann Rheum Dis 66: 87-90.

18. Romagnani S, Maggi E, Liotta F, et al. (2009): Properties and origin of human Th17 cells. Mol Immunol 47: 3-7.

19. Louten J, Boniface K, de Waal Malefyt R (2009): Development and function of Th17 cells in health and disease. J Allergy Clin Immunol 123: 1004-1011.

20. Ghilardi N, Ouyang W (2007): Targeting the development and effector functions of Th17 cells. Semin Immunol 19: 383-393.

21. Fuss IJ, Heller F, Boirivant M, et al. (2004): Non classical CD1d-restricted NK T cells that produce IL-13 characterize an atypical Th2 response in ulcerative colitis. J Clin Invest 113: 1490-1497.

22. Jiang W, Su J, Zhang X, et al. (2014): Elevated levels of Th17 cells and Th17-related cytokines are associated with disease activity in patients with inflammatory bowel disease. Inflamm Res 63: 943-950.

23. Lohr J, Knoechel B, Caretto D, Abbas AK (2009): Balance of Th1 and Th17 effector and peripheral regulatory T cells. Microbes Infect 11: 589-593.

24. Zorzi F, Monteleone I, Sarra M, et al. (2013): Distinct Profiles of Effector Cytokines Mark the Different Phases of Crohn's Disease. PLoS One 8: e54562.

25. Ogino T, Nishimura J, Barman S, et al. (2013): Increased Th17-inducing activity of CD14+ CD163 low myeloid cells in intestinal lamina propria of patients with Crohn's disease. Gastroenterology 145: 1380-1391.

26. Xavier RJ, Podolsky DK (2007): Unravelling the pathogenesis of inflammatory bowel disease. Nature 448: 427-434.

27. Vucelic B i saradnici. (2002): Gastroenterologija i hepatologija. I edicija. Zagreb.

28. Sarto RB (2006): Mechanisms of Disease: Pathogenesis of Crohn's Disease and Ulcerative Colitis. Nat Clin Pract Gastroenterol Hepatol 3: 390-407.

29. Szczeklik K, Owczarek D, Pytko-Polończyk J (2012): Proinflammatory cytokines in the saliva of patients with active and nonactive Crohn's disease. Pol Arch Med Wewn 122: 200-208.

30. Solem CA, Loftus EV, Tremaine WJ, et al. (2005): Correlation of C-reactive protein with clinical, endoscopic, histologic and radiographic activity in inflammatory bowel disease. Inflammatory Bowel Diseases 11: 707-712. 
31. Schreiber S, Heinig T, Thiele HG, Raedler A (1995): Immunoregulatory role of interleukin 10 in patients with inflammatory bowel disease. Gastroenterology 108: 1434-1444.

32. Fujino S, Andoh A, Bamba S, et al. (2003): Increased expression of interleukin 17 in inflammatory bowel disease. Gut 52: 65-70.

33. Yen D, Cheung J, Scheerens H, et al. (2006): IL-23 is essential for $\mathrm{T}$ cell-mediated colitis and promotes inflammation via IL-17 and IL-6. J Clin Invest 116: 1310-1316.

34. Fuss IJ, Heller F, Boirivant M, et al. (2004): Nonclassical CD1d-restricted NK T cells that produce IL-13 characterize an atypical Th2 response in ulcerative colitis. J Clin Invest 113: 1490-1497.

35. Hanauer SB (1996): Inflammatory bowel disease. N Engl J Med 334: 841-848.

36. Fatahzadeh M (2009): Inflammatory bowel disease. Oral Surg Oral Med Oral Radiol Endod 108: e1-e10.

37. Greenstein AJ, Janowitz HD, Sachar DB (1976): The extra-intestinal complications of Crohn's disease and ulcerative colitis: a study of 700 patients. Medicine 55: 401-412.

38. Scheper HJ, Brand HS (2009): Oral aspects of Crohn's disease. Int Dent J 52: 163-172.

39. Galbraith SS, Drolet BA, Kugathasan S, et al. (2005): Asymptomatic inflammatory bowel disease presenting with mucocutaneous findings. Pediatrics 116: 439-445.

40. Dupuy A, Cosnes J, Revuz J, et al. (1999): Oral Crohn's disease: clinical characteristics and long term follow up of 9 cases. Arch Dermatol 135: 439-442.

41. Graham DB, Jager DL, Borum ML (2006): Metastatic Crohn's disease to the face. Dig Dis Sci 51: 2062-2063.

42. Storwick GS, Prihoda MB, Fulton RJ, Wood WS (1994): Pyodermatitis-pyostomatitis vegetans: a specific marker for inflammatory bowel disease. J Am Acad Derm 31: 336-341.

43. Chan SW, Scully C, Prime SS, Eveson J (1991): Pyostomatitis vegetans: oral manifestation of ulcerous colitis. Oral Surg Oral Med Oral Pathol 72: 689-692.

44. Hansen LS, Silverman S Jr, Daniels TE (1983): The differential diagnosis of pyostomatitis vegetans and its relation to bowel disease. Oral Surg Oral Med Oral Pathol 55: 363-373.

45. Jarrett P, Duffill M, Oakley A, Smith A (1997): Pellagra, azathiorpine and inflammatory bowel disease. Clin Exp Derm 22: 44-45.

46. Oliva MM, Lake AM (1996): Nutritional considerations and management of the child with inflammatory bowel disesase. Nutrition 12: 151-158.

47. Franch AM, Soriano YJ, Sarrion Perez MG (2010): Dental management of patients with inflammatory bowel disease. J Clin Exp Dent 2: e191-5.

48. Szczeklik K, Owczarek D, Pytko-Polończyk J, et al. (2012): Proinflammatory cytokines in the saliva of patients with active and nonactive Crohn's disease. Pol Arch Med Wewn 122: 200-208.

49. Aleksandra Nielsen A, Niderby Nielsen J, Schmedes A, et al. (2005): Saliva interleukin-6 in patients with inflammatory bowel disease. Scand J Gastroenterol 40: 1444-1448.

50. Zhang Y, Lin M, Zhang S, et al. (2008): NF-kappaB-dependent cytokines in saliva and serum from patients with oral lichen planus: a study in an ethnic Chinese population. Cytokine 41: 144-149.

51. Rhodus NL, Ho V, Miller CS, et al. (2005): NF-kappaB dependent cytokine levels in saliva of patients with oral preneoplastic lesions and oral squamous cell carcinoma. Cancer Detect Prev 29: 42-45.
52. Rezaie A, Khalaj S, Shabihkhani M, et al. (2006): Study on the Correlations among Disease Acttivity Index and Salivary Transforming Growth Factor- $\beta 1$ and Nitric Oxide in Ulcerative Colitis Patients. Ann N Y Acad Sci 4: 17-21.

53. Grossner-Schreiber B, Fetter T, Hedderich J, et al. (2006): Prevalence of dental caries and periodontal disease in patients with inflammatory bowel disease: a case control study. J Clin Periodontol 33: 478-484.

54. Brito F, de Barros FC, Zaltman C, et al. (2008): Prevalence of periodontitis and DMFT index in patients with Crohn's disease and ulcerative colitis. J Clin Periodontol 35: 555-560.

55. Habashneh RA, Khader YS, Alhumouz MK (2012): The association between inflammatory bowel disease and periodontitis among Jordanians: a case-control study. J Periodontal Res 47: 293-298.

56. Sundh B, Emilson CG (1989): Salivary and microbial conditions and dental health in patients with Crohn's disease: a 3 year study. Oral Surg Oral Med Oral Pathol 67: 286-290.

57. Szymanska S, Lordal M, Rathnayake N, et al. (2014): Dental caries, prevalence and risk factors in patients with Crohn's disease. PLoS One 9: e91059.

58. Singhal S, Dian D, Keshavarzian A, et al. (2011): The role of oral hygiene in inflammatory bowel disease. Dig Dis Sci 56: 170-175.

59. Stein JM, Lammert F, Zimmer V, et al. (2010): Clinical Periodontal and Microbiologic Parameters in Patients With Crohn's Disease With Consideration of the CARD15 Genotype. J Periodontol 81: 535-545.

60. Oz HS, Chen T, Etherhole JL (2010): A model for chronic mucosal inflammation in IBD and periodontitis. Dig Dis Sci 55: 2194-2202.

61. Indriolo A, Greco S, Ravelli P, Fagiuoli S (2011): What can we learn about biofilm/host interactions from the study of inflammatory bowel disease. J Clin Periodontol 38 (Suppl 11): 36-43.

62. Garlet GP, Matrins WJr, Fonseca BA, et al. (2004): Matrix metalloproteinases, their physiological inhibitors and osteoclast factors are differentially regulated by the cytokine profile in human periodontal disease. J Clin Periodontol 31: 671-679.

63. Pizarro TT, Cominelli F (2007): Cytokine therapy for Crohn's disease. Advances in translational research Ann Rev Med 58: 433-444.

64. Rezaie A, Khalaj S, Shabihkhani M, et al. (2006): Study on the Correlations among Disease Acttivity Index and Salivary Transforming Growth Factor- $\beta 1$ and Nitric Oxide in Ulcerative Colitis Patients. Ann N Y Acad Sci 4: 17-21.

65. Waddington RJ, Moseley R, Embery G (2000): Periodontal Disease Mechanisms: Reactive oxygen species: a potential role in the pathogenesis of periodontal diseases. Oral Dis 6: 138-151.

66. Figueredo CM, Brito F, Barros FC, et al. (2011): Expression of cytokines in the gingival crevicular fluid and serum from patients with inflammatory bowel disease and untreated chronic periodontitis. J Periodontal Res 46: 141-146.

67. Salvi GE, Brown CE, Fujihashi K, et al. Inflammatory mediators of the terminal dentition in adult and early onset periodontitis. J Periodont Res 1998; 33: 212-225.

68. Schutz T, Drude C, Paulisch E, et al. (2003): Sugar intake, taste changes and dental health in Crohn's disease. Dig Dis 21: 252257.

69. Jarnerot G, Jarnmark I, Nilsson K (1983): Consumption of refined sugars by patients with Crohn's disease, ulcerative colitis or irritable bowel syndrome. Scand J Gastroent 18: 999-1002. 
70. Marlow GJ, van Gent D, Ferguson LR (2013): Why interleukin-10 supplementation does not work in Crohn's disease patients. World J Gastroenterol 19: 3931-3941.

71. Sundh B, Hulden L (1982): Oral status in patients with Crohn's disease. Acta Chi Scand 148: 531-534.

72. Han YW, Wang X (2013): Mobile Microbiome: Oral Bacteria in Extra-oral Infections and Inflammation. Dent Res 92: 485491.

73. Lockhart PB, Brennan MT, Sasser HC, et al. (2008): Bacteriemia associated with toothbrushing and dental extraction. Circulation 117: 3118-3125.

74. Naka S, Nomura R, Takashima Y, et al. (2014): A specific Streptococcus mutans strain aggravates non-alcoholic fatty liver disease. Oral Dis 20: 700-706.

75. Kojimra A, Nakano K, Wada K, et al. (2012): Infection of specific strains of Streptococcus mutans, oral bacteria, confers a risk of ulcerative colitis. Nature Sci Reports 2: doi 10.1038/ srep00332.

76. Strauss J, Kaplan GG, Beck PL, et al. (2011): Invasive potential of gut mucosa-derived Fusobacterium nucleatum positively correlates with IBD status of the host. Infl Bow Dis 17: 19711978.

77. Ismail Y, Mahendran V, Ocavia S, et al. (2012): Investigation of the enteric pathogenic potential of oral Campylobacter concisus strains isolated from the patients with inflammatory bowel disease. PLoS One 7: e38217.

78. Nakano K, Nomura R, Hemoto H, et al. (2007): Detection of novel serotype $\mathrm{k}$ Streptococcus mutans in infective endocarditis patients. J Med Microbiol 56: 1413-1415.

79. Nakano K, Nomura R, Nakagawa I, et al. (2004): Demonstration of Streptococcus mutans with a cell wall polysaccharide specific to a new serotype, $\mathrm{k}$, in the human oral cavity. J Clin Microbiol 42: 198-202.

80. Schreiber S, Fedorak RN, Nielsen OH, et al. (2000): Safety and efficacy of recombinant human interleukin 10 in chronic active Crohn's disease. Gastroenterology 119: 1461-1472.

81. Bianchi ML (2010): Inflammatory bowel diseases, celiac disease and bone. Arch Biochem Biophys 503: 54-65.

82. Harris L, Senagore P, Young VB, McCabe IR (2009): Inflammaoty bowel disease causes reversible supression of osteoblast and chondrocyte function in mice. Am J Phys Gast Liver Phys 296: G1020-G1029.

83. Alsaadi G, Quirynen M, Komarek A, van Steenberghe D (2007): Impact of local and systemic factors on the incidence of oral implant failures up to abutment connection. J Clin Periodont 34: 610-617.

84. Alsaadi G, Quirynen M, Michiles K, et al. (2008): Impact of local and systemic factors on the incidence of oral implant failures up to abutment connection with modified surface oral implants. J Clin Periodont 35: 51-57.

85. Kuchler U, Luvizuto ER, Munoz F, et al. (2013): Bone healing around titanium impalnts in two rat colitis models. Clin Oral Imp Res 24: 224-229.

86. Thurston RD, Larmonier CB, Majewski PM (2010): Tumor necrosis factor and interferon $\gamma$ down regulate Klotho in mice with colitis. Gastroenterology 138: 1384-1394.

87. Rowland M, Fleming P, Bourke B (2010): Looking in the mouth for Crohn's disease. Inflamm Bowel Dis 16: 332-337.

88. Bhavsar M, Amiji M (2008): Oral IL-10 gene delivery in a microsphere-based formulation for local transfection and therapeutic efficacy in inflammatory bowel disease. Gene Ther 15: 1200-1209.
89. Sales-Campos H, Basso PJ, Alves VB, et al. (2014): Classical and recent advances in the treatment of inflammatory bowel diseases. Braz J Med Biol Res 48: 96-107.

90. D'Haens GR, Sartor RB, Silverberg MS, et al. (2014): Future directions in inflammatory bowel disease management. J Crohns Colitis 8: 726-734.

91. Galbraith SS, Drolet BA, Kugathasan S, et al. (2005): Asymptomatic inflammatory bowel disease presenting with mucocutaneous findings. Pediatrics 116: 439-445.

92. Armuzzi A, Lionetti P, Blandizzi C, et al. (2014): Anti-TNF agents as therapeutic choice in immune-mediated inflammatory diseases: focus on adalimumab. Int J Immunopathol Pharmacol 27: 11-32.

93. Keane J, Gershon S, Wise RP, et al. (2001): Tuberculosis associated with infliximab, a tumor necrosis factor alpha-neutralizing agent. N Engl J Med 345: 1098-1104.

94. Hueber W, Sands BE, Lewitzky S, et al. (2012): Secukinumab, a human anti-IL-17A monoclonal antibody, for moderate to severe Crohn's disease: unexpected results of a randomised, double-blind placebo-controlled trial. Gut 61: 16931700.

95. Perrier C, Rutgeerts P (2011): Cytokine blockade in inflammatory bowel diseases. Immunotherapy 3: 1341-1352.

96. Van Deventer SJ, Elson CO, Fedorak RN (1997): Multiple doses of intravenous interleukin 10 in steroid-refractory Crohn's disease. Crohn's Disease Study Group. Gastroenterology 113: 383-389.

97. Colombel JF, Rutgeerts P, Malchow H, et al. (2001): Interleukin 10 (Tenovil) in the prevention of postoperative recurrence of Crohn's disease. Gut 49: 42-46.

98. Fedorak RN, Gangl A, Elson CO, et al. (2000): Recombinant human interleukin 10 in the treatment of patients with mild to moderately active Crohn's disease. Gastroenterology 119: 1473-1482.

99. Herfarth H, Schölmerich J (2002): IL-10 therapy in Crohn's disease: at the crossroads. Treatment of Crohn's disease with the anti-inflammatory cytokine interleukin 10 . Gut 50: 146147.

100. Rachmawati H, Beljaars L, Reker-Smit C, et al. (2004): Pharmacokinetic and biodistribution profile of recombinant human interleukin-10 following intravenous administration in rats with extensive liver fibrosis. Pharm Res 21: 2072-2078.

101. Barbara G, Xing Z, Hogaboam CM, et al. (2000): Interleukin 10 gene transfer prevents experimental colitis in rats. Gut 46 : 344-349.

102. Del Carmen S, Zurita-Turk M, Alvarenga Lima F, et al. (2013): A novel interleukin-10 DNA mucosal delivery system attenuates intestinal inflammation in a mouse model. Eur J Inflamm 11: 641-645.

103. Del Carmen S, Martín Rosique R, Saraiva T, et al. (2014): Protective effects of lactococci strains delivering either IL-10 protein or cDNA in a TNBS-induced chronic colitis model. J Clin Gastroenterol 48 Suppl 1: S12-17.

104. Vavricka SR, Manser CN, Hediger S, et al. (2013): Periodontitis and gingivitis in inflammatory bowel disease: a case-control study. Inflamm Bowel Dis 19: 2768-2777.

105. Siegel MA, Jacobson JJ (1999): Inflammatory bowel disease and the oral cavity. Oral Surg Oral Med Oral Pathol Endod 87: $12-14$.

106. Sartor RB (2008): Microbial influences in inflammatory bowel diseases. Gastroenterol 134: 577-594. 